\title{
Front Matter: Volume 7630
}

, "Front Matter: Volume 7630," Proc. SPIE 7630, Passive Components and Fiber-based Devices VI, 763001 (10 December 2009); doi:

$10.1117 / 12.852900$

SPIE Event: Asia Communications and Photonics, 2009, Shanghai, Shanghai , SPlE. China 


\title{
PROCEEDINGS OF SPIE
}

\section{Passive Components and Fiber-based Devices VI}

\author{
Perry P. Shum \\ Editor \\ 2-6 November 2009 \\ Shanghai, China \\ Cosponsored by \\ Optical Society of America \\ IEEE Photonics Society \\ SPIE \\ Chinese Optical Society \\ China Institute of Communications \\ Local Organizing Committee \\ Shanghai Jiao Tong University \\ Shanghai Institute of Optics and Fine Mechanics \\ Alcatel-Lucent \\ Fudan University \\ Published by \\ SPIE \\ Optical Society of America \\ IEEE Photonics Society
}

Volume 7630 
The papers included in this volume were part of the technical conference cited on the cover and title page. Papers were selected and subject to review by the editors and conference program committee. Some conference presentations may not be available for publication. The papers published in these proceedings reflect the work and thoughts of the authors and are published herein as submitted. The publisher is not responsible for the validity of the information or for any outcomes resulting from reliance thereon.

Please use the following format to cite material from this book:

Author(s), "Title of Paper," in Passive Components and Fiber-based Devices Vl, edited by Perry P. Shum, Proceedings of SPIE-OSA-IEEE Asia Communications and Photonics Vol. 7630 (SPIE, Bellingham, WA, 2009) Article CID Number.

ISSN 0277-786X

ISBN 9780819480323

Published by

SPIE

P.O. Box 10, Bellingham, Washington 98227-0010 USA

Telephone +1 3606763290 (Pacific Time) · Fax +1 3606471445

SPIE.org

Optical Society of America

2010 Massachusetts Ave., N.W., Washington, D.C., 20036 USA

Telephone +1 2022238130 (Eastern Time) · Fax +1 2022231096

OSA.org

IEEE Photonics Society

445 Hoes Lane, Piscataway, New Jersey, 08855 USA

Telephone +1 7325628434 (Eastern Time) · Fax +1 7325628434

IEEE.org

Copyright (c) 2009, Society of Photo-Optical Instrumentation Engineers, Optical Society of America, and IEEE Photonics Society.

Copying of material in this book for internal or personal use, or for the internal or personal use of specific clients, beyond the fair use provisions granted by the U.S. Copyright Law is authorized by SPIE subject to payment of copying fees. The Transactional Reporting Service base fee for this volume is $\$ 18.00$ per article (or portion thereof), which should be paid directly to the Copyright Clearance Center (CCC), 222 Rosewood Drive, Danvers, MA 01923. Payment may also be made electronically through CCC Online at copyright.com. Other copying for republication, resale, advertising or promotion, or any form of systematic or multiple reproduction of any material in this book is prohibited except with permission in writing from the publisher. The CCC fee code is $0277-786 \mathrm{X} / 09 / \$ 18.00$.

Printed in the United States of America.

Publication of record for individual papers is online in the SPIE Digital Library.

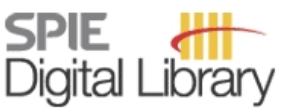

SPIEDigitalLibrary.org

Paper Numbering: Proceedings of SPIE follow an e-First publication model, with papers published first online and then in print and on CD-ROM. Papers are published as they are submitted and meet publication criteria. A unique, consistent, permanent citation identifier (CID) number is assigned to each article at the time of the first publication. Utilization of CIDs allows articles to be fully citable as soon they are published online, and connects the same identifier to all online, print, and electronic versions of the publication. SPIE uses a six-digit CID article numbering system in which:

- The first four digits correspond to the SPIE volume number.

- The last two digits indicate publication order within the volume using a Base 36 numbering system

employing both numerals and letters. These two-number sets start with 00, 01, 02, 03, 04, 05, 06, 07, 08, 09, 0A, $\mathrm{OB}$... OZ, followed by 10-1Z, 20-2Z, etc.

The CID number appears on each page of the manuscript. The complete citation is used on the first page, and an abbreviated version on subsequent pages. Numbers in the index correspond to the last two digits of the six-digit CID number. 


\title{
Contents
}

\author{
xiii Organizing Committee
}

xiv Conference Committee

\section{BEST STUDENT PAPER COMPETITION}

763002 Optical time-slot swapping based on parametric wavelength exchange [7630-74]

$X . X U$, The Univ. of Hong Kong (Hong Kong, China); M. Shen, Univ. of British Columbia (Canada); T. I. Yuk, K. K. Y. Wong, The Univ. of Hong Kong (Hong Kong, China)

763003 Phase-tunable polarization division multiplexing DPSK receiver using a single demodulator [7630-99]

Y. Gao, Y. Xie, Zhejiang Univ. (China); L. Xu, NEC Labs. America (United States); S. He,

Zhejiang Univ. (China)

763004 Refractive index sensor with acoustic grating in a low index contrast photonic bandgap fiber [7630-100]

Q. Shi, Nankai Univ. (China) and Univ. of Sydney (Australia); B. Kuhlmey, D. Wu, Univ. of Sydney (Australia)

763005 Simple technique for measuring Raman gain efficiency spectrum distribution in a single-mode fiber [7630-118]

Y. Tsutsumi, M. Ohashi, Osaka Prefecture Univ. (Japan)

763006 Long distance fiber Bragg grating sensor system based on erbium-doped fiber and Raman amplification [7630-86]

J. Hu, National Univ. of Singapore (Singapore); Z. Chen, X. Yang, J. Ng, A-STAR Institute for Infocomm Research (Singapore); C. YU, National Univ. of Singapore (Singapore) and A-STAR Institute for Infocomm Research (Singapore)

763007 Logic unit for CSRZ-OOK signals with the capability of simultaneously realizing logic OR and AND gates (Best Student Paper Award) [7630-06]

B. WU, J. WU, L. Li, K. XU, X. Hong, J. Lin, Beijing Univ. of Posts and Telecommunications (China)

\section{OPTICAL FIBER}

763008 Brillouin dynamic grating in optical fibers and its applications (Invited Paper) [7630-120]

K. Y. Song, Chung-Ang Univ. (Korea, Republic of)

$76300 \mathrm{~A}$ Investigation on temperature sensitivity of D-shaped fibers by manufacturing specified surface structure [7630-121]

O.-J. Kwon, H.-J. Kim, S. Chu, S. H. Song, Y.-G. Han, Hanyang Univ. (Korea, Republic of)

$7630 \mathrm{OB}$ High temperature sensing characteristics of erbium-doped fiber using fluorescence intensity ratio technology [7630-03]

J. Peng, L. Liu, Y. Fu, H. Wei, J. Wang, S. Jian, Beijing Jiaotong Univ. (China) 
7630 OC A novel scheme for tunable optical pulse generation based on fiber sagnac loop [7630-50] W. Yang, Y. Yang, P. Zhang, M. Yang, Beihang Univ. (China)

7630 OD A $100 \mathrm{GHz}$ pulse train generation by spectral filtering of a $10 \mathrm{GHz}$ mode-locked fiber laser output [7630-90]

J. Zhang, W.-J. Seo, I.-H. Cho, D.-S. Seo, Myongji Univ. (Korea, Republic of)

7630 OE Propagation of high-power parabolic pulses in cubicon fiber amplifiers [7630-83]

S. Zhang, C. Jin, Y. Meng, Hebei Normal Univ. (China)

\section{POLARIZATION EFFECTS AND MEASUREMENTS}

7630 OF Experimental demonstration of a wavelength tunable polarization OTDR using an SOA for received signal amplification [7630-88]

I. Yamashita, Kansai Electric Power (Japan); M. Ohashi, Osaka Prefecture Univ. (Japan)

7630 OG Design of broadband single-polarization single-mode holey fiber [7630-80]

T. Guo, S. Lou, Y. Lu, H. Li, L. Wang, W. Chen, Beijing Jiaotong Univ. (China)

$7630 \mathrm{OH}$ An experiment of polarization measurement using DSP-based control system [7630-116] X. Weng, X. Zhao, X. Yuan, F. Tian, X. Zhang, Beijing Univ. of Posts and Telecommunications (China)

\section{PHOTONIC CRYSTALS}

7630 ol The designs of $4 \times 2$ encoder based on photonic crystals [7630-95] K.-Y. Lee, China Univ. of Science and Technology (Taiwan, China); Y.-C. Yang, National Taipei Univ. of Technology (Taiwan, China); Y.-J. Lin, W.-Y. Lee, C.-L. Lee, China Univ. of Science and Technology (Taiwan, China); S.-H. Wong, National Taiwan Univ. (Taiwan, China)

7630 0J Folded Mach-Zehnder interferometer based on photonic crystal self-collimation effect [7630-31]

X. Chen, Minjiang Univ. (China); Y. Wang, Longyan Univ. (China); S. Chen, X. Li, N. Lin, G. Lin, Minjiang Univ. (China); B. Ni, J. Bai, J. Li, Z. Qiang, Fujian Normal Univ. (China)

7630 OK Terahertz waveguides based on photonic crystal [7630-107]

J. Li, X. Zhao, J. Li, China Jiliang Univ. (China)

$7630 \mathrm{OL} \quad$ Asymmetrical interleaver filters based on one-dimensional photonic crystal theory [7630-63] S. YU, J. Zhang, Shanghai Univ. (China)

FIBER LASERS

7630 OM 32Tb/s DWDM Transmission System (Invited Paper) [7630-101]

J. Yu, NEC Labs. America, Inc. (United States); X. Zhou, AT\&T Labs. Research (United States) 
7630 ON Influence of pump power on the output characteristics of multiwavelength erbium-doped fiber laser employing symmetrical nonlinear optical loop mirror [7630-34]

Y. Sun, J. Tian, Y. Yao, X. YU, D. Chen, Harbin Institute of Technology (China)

763000 Passive harmonically mode-locked erbium-doped fiber laser [7630-01]

Z. Zhang, Q. Kuang, M. Sang, Jiangxi Normal Univ. (China)

7630 OP Q-switched Yb-doped microstructure fiber laser using GaAs as saturable absorber [7630-97] S. Fu, X. Liu, Shandong Univ. of Technology (China)

$76300 Q$ Characterization of fused-silica optical fibers delivery high-peak power YAG laser beam [7630-16]

X. Zhao, Y. Gao, Chinese Academy of Engineering Physics (China)

\section{OPTICAL PROCESSING}

7630 OR Spectral phase OCDMA encoder/decoder using travelling interference fringe photo-writing technique [7630-103]

I. Fsaifes, Univ. de Limoges (France) and Univ. de Lille (France); A. Millaud, 3S Photonics (France) and Univ. de Lille (France); S. Cordette, Télécom ParisTech (France); C. Lepers, Télécom SudParis (France); M. Douay, Univ. de Lille (France); C. Ware, Télécom ParisTech (France)

7630 OS Receiver sensitivity improvement for NRZ-OOK signal by optical parametric amplifier-assisted detection [7630-53]

Y. Liang, P. C. Chui, K. K. Y. Wong, The Univ. of Hong Kong (Hong Kong, China)

FIBER GRATINGS

7630 oU Polarization sensitivities of demodulation techniques for tilted fiber Bragg grating refractometer [7630-24]

Y.-C. Lu, Beijing Jiaotong Univ. (China); W.-P. Huang, McMaster Univ. (Canada); S.-S. Jian, Beijing Jiaotong Univ. (China)

$76300 \mathrm{~V}$ The experimental fabrication of add/drop filters using a Bragg grating-assisted mismatched coupler [7630-38]

L. Y. Fan, W. W. Jiang, Beijing Jiaotong Univ. (China); J. Li, Beijing Jiaotong Univ. (China) and China Academy of Railway Sciences (China); J. J. Zheng, Z. M. Liu, S. S. Jian, Beijing Jiaotong Univ. (China)

\section{OPTICAL COUPLERS}

7630 OW Bragg grating-assisted optical triplexer using two silicon nanowire-based directional couplers [7630-27]

N. Zhu, Z. Wang, L. Wosinski, Royal Institute of Technology (Sweden) and Zhejiang Univ. (China); S. He, Royal Institute of Technology (Sweden)

7630 0X Index profile engineering of multimode interference couplers [7630-200]

A. Ortega-Moñux, I. Molina-Fernández, J. G. Wangüemert-Pérez, Univ. de Málaga (Spain) 
7630 OY Modified Hamming function weighted waveguide structure for the broadband and minimized mismatched optical couplers [7630-203]

C.-F. Chen, Y.-S. Ku, National Central Univ. (Taiwan, China); T.-T. Kung, National Central Univ. (Taiwan, China) and National United Univ. (Taiwan, China); H.-W. Wang, Industrial Technology Research Institute (Taiwan, China); M.-S. Yeh, Chung-Shan Institute of Science and Technology (Taiwan, China)

$76300 Z$ Low-crosstalk, short-length mismatched optical coupler designed by new weighting function [7630-204]

C.-F. Chen, National Central Univ. (Taiwan, China)

763010 Silicon wires and compact multi-mode interference splitters with an uneven splitting ratio [7630-40]

J. Zhou, H. Shen, H. Zhang, X. Liu, Institute of Microelectronics (China)

763011 Analysis and fabrication of broadband add/drop filters using a Bragg grating-assisted mismatched coupler [7630-39]

L. Y. Fan, Beijing Jiaotong Univ. (China); J. Li, Beijing Jiaotong Univ. (China) and China Academy of Railway Sciences (China); W. W. Jiang, J. J. Zheng, Z. M. Liu, S. S. Jian, Beijing Jiaotong Univ. (China)

\section{PHOTONIC CRYSTAL FIBERS I}

763012 Ultra-wideband single-polarization single-mode photonic crystal fiber with high nonlinearity and low dispersion [7630-108]

L. An, Z. Zheng, Z. Li, Y. Liu, Beihang Univ. (China); T. Zhou, New Jersey Institute of Technology (United States); J. Cheng, The Pennsylvania State Univ. (United States)

763013 A novel proposal for DWDM demultiplexer design using resonance cavity in photonic crystal structure [7630-58]

A. Rostami, Univ. of Tabriz (Iran, Islamic Republic of); H. Habibiyan, Amirkabir Univ. of Technology (Iran, Islamic Republic of); F. Nazari, A. Bahrami, H. Alipour Banaei, Univ. of Tabriz (Iran, Islamic Republic of)

\section{OPTICAL WAVEGUIDE DEVICES I}

763014 Signal processing in silicon waveguides (Invited Paper) [7630-202] Y. SU, F. Liu, Q. Li, Q. Chang, Shanghai Jiao Tong Univ. (China); Z. Zhang, M. Qiu, Royal Institute of Technology (Sweden)

763015 Design of suspended SU-8 optical waveguides for ultrasmall bending [7630-85]

B. Yang, Z. Sheng, D. Dai, Zhejiang Univ. (China)

763016 Polarization-insensitive electro-optical modulator based on polymer-filled silicon cross-slot waveguide [7630-93]

W. Wang, H. Zhou, J. Yang, M. Wang, X. Jiang, Zhejiang Univ. (China)

763017 Analysis and design of box-like filters based on $3 \times 2$ microring resonator arrays [7630-81] X. Zhang, Shanghai Univ. (China); X. Zhang, D. Huang, Huazhong Univ. of Science and Technology (China) 
763018 Integrated optical microwave channeliser [7630-104]

M. W. Austin, RMIT Univ. (Australia)

FIBER DESIGN AND FABRICATION

763019 Fabrication and design of asymmetrical twin core fiber for passive mode-locking [7630-28]

L. Yao, S. Lou, L. Liu, J. Peng, S. Jian, Beijing Jiaotong Univ. (China)

7630 1A Theoretical design of low-loss single-polarization single-mode microstructured polymer optical Fiber [7630-05]

Y. Zhang, Xi'an Institute of Optics and Precision Mechanics (China) and Baoji College of Arts and Science (China); X. Li, Y. Gong, L. Wang, Xi'an Institute of Optics and Precision Mechanics (China)

\section{OPTICAL WAVEGUIDE DEVICES II}

7630 1B Spectroscopic properties of $\mathrm{Tm}^{3+}$ ions in $\mathrm{Tm}^{3+} / \mathrm{Yb}^{3+}$ codoped tellurite glass [7630-91] Q. Huang, Q. Wang, J. Chang, X. Zhang, Shandong Univ. (China); Z. Liu, National Univ. of Defense Technology (China)

7630 1C Cu-Na ion exchange soda-lime glass planar waveguides and their photoluminescence [7630-48]

Y. Ti, X. He, J. Zhang, J. Zheng, Jilin Univ. (China); P. Wang, G. Farrell, Dublin Institute of Technology (Ireland)

7630 ID Variable optical power splitter based on slot waveguide [7630-02]

L. Zheng, M. Zhu, Jinan Univ. (China)

$76301 \mathrm{E} \quad$ Design of athermal all-polymer waveguide microring resonator [7630-13] X. Han, M. Zhao, J. Zhang, L. Wang, J. Teng, J. Wang, X. Jian, Dalian Univ. of Technology (China)

\section{PHOTONIC CRYSTAL FIBERS II}

$7630 \mathrm{IF} \quad$ Ultra-flattened chromatic dispersion photonic crystal fibers with high nonlinearity for supercontinuum generation [7630-67]

Y. Wang, X. Zhang, X. Ren, L. Zheng, X. Liu, Y. Huang, Beijing Univ. of Posts and Telecommunications (China)

7630 1G Slope-matching profile optimization of dual-concentric-core photonic crystal fiber for broadband dispersion compensation [7630-49]

J. Han, S. Hou, Lanzhou Univ. of Technology (China)

$7630 \mathrm{1H} \quad$ Two-mode photonic crystal fiber interferometer for temperature and strain sensing [7630-21] H. Li, S. Lou, S. Feng, T. Guo, L. Wang, W. Chen, S. Jian, Beijing Jiaotong Univ. (China)

763011 Spectral compression of femtosecond pulses in photonic crystal fiber with anomalous dispersion [7630-105]

H. P. Li, X. J. Zhang, J. K. Liao, X. G. Tang, Y. Liu, Y. Z. Liu, Univ. of Electronic Science and Technology of China (China) 
$7630 \mathrm{lJ}$ Second-order effects in fiber optical parametric amplifier (Invited Paper) [7630-92] K. K. Y. Wong, The Univ. of Hong Kong (Hong Kong, China)

$7630 \mathrm{lK}$ Multi-tap photonic microwave filter based on two-pump fiber optical parametric amplifier [7630-82]

J. Li, K. K. Y. Cheung, X. XU, K. K. Y. Wong, The Univ. of Hong Kong (Hong Kong, China)

$7630 \mathrm{lL} \quad$ Experimental research on secondary pulsation in an all-fiber pulsed amplifier [7630-47] W. Li, K. Tao, Z. Wang, Y. Liu, Nankai Univ. (China)

$76301 \mathrm{M}$ A new adaptive erbium-doped fiber amplifier [7630-68] T. Xiao, S. Xiao, H. Chen, L. Ge, D. Ding, Shanghai Jiao Tong Univ. (China); Z. Liu, The Chinese Univ. of Hong Kong (Hong Kong, China); C. Yang, Shanghai Jiao Tong Univ. (China)

\section{OPTICAL DEVICES 1}

7630 iN A novel FBG laser sensor based on beat frequency modulation technology [7630-72] L. Zhang, Nanjing Univ. (China); S. Liu, Heilongjiang Univ. (China) and Nanjing Univ. (China); Z. Yin, L. Gao, X. Chen, Nanjing Univ. (China)

763010 Metal-based $1 \times 2$ plastic optical fiber (POF) splitter for video over POF system application [7630-102] A. A. Ehsan, S. Shaari, Univ. Kebangsaan Malaysia (Malaysia); M. K. Abd Rahman, Univ. Teknologi MARA (Malaysia)

7630 IP Eight-channel wavelength division demultiplexer using multimode interference [7630-54] A. Rostami, A. Bahrami, F. Nazari, H. Alipour Banaei, Univ. of Tabriz (Iran, Islamic Republic of)

$76301 Q \quad$ Investigation of a Fabry-Perot-based optical filter for broadband multichannel communication systems [7630-30]

J. Zhang, CETC22 (China); Y. Guo, J. Huo, G. Wang, S. Zhang, Jilin Univ. (China)

$76301 \mathrm{R}$ Compact in-fiber Mach-Zehnder interferometer using a twin-core fiber [7630-11] S. Feng, H. Li, O. XU, S. LU, S. Jian, Beijing Jiaotong Univ. (China)

\section{OPTICAL FIBER}

7630 is Progress in semiconductor optical fibers (Invited Paper) [7630-201]

J. Ballato, T. Hawkins, P. Foy, B. Kukuoz, C. McMillen, R. Stolen, Clemson Univ. (United States); N. K. Hon, B. Jalali, Univ. of California, Los Angeles (United States); R. Rice, Northrop Grumman Space Technology (United States)

7630 IT The improvement of transmission properties for multi-mode fiber based on launching optical field shaping and feedback equalization [7630-78]

W. Fang, J. Zhang, Y. Shao, B. Huang, N. Chi, Fudan Univ. (China) 
$76301 \mathrm{U}$ Wave breaking in dispersion-decreasing fiber with normal group-velocity dispersion [7630-64]

G. Xia, L. Liu, S. Li, L. Zhou, D. Li, Wuhan Univ. of Science and Engineering (China)

$76301 \mathrm{~V}$ Longitudinal fiber parameter measurements of pure silica core fibers based on OTDR technique [7630-119]

M. Ohashi, Y. Tsutsumi, Osaka Prefecture Univ. (Japan); I. Yamashita, Kansai Electric Power (Japan)

\section{OPTICAL DEVICES II}

7630 IW Two- and three-dimensional studies of a silicon-based chromatic dispersion compensator [7630-94]

C. E. J. Png, S. T. Lim, E. P. Li, A-STAR (Singapore); A. J. Danner, National Univ. of Singapore (Singapore); K. Ogawa, Y. T. Tan, Fujikura Ltd. (Japan)

$76301 \mathrm{X}$ Optimal design of cascaded long-period waveguide grating equalizer for broadband Er-Yb co-doped phosphate glass waveguide amplifiers [7630-09]

H. Chen, Yangtze Univ. (China)

7630 IY Low-loss bend-bend coupler for an ultra-small microring resonator based on Si nanowires [7630-89]

J. Hu, D. Dai, Zhejiang Univ. (China)

\section{POSTER SESSION}

763012 The dispersion and dispersion slope characteristics of the fiber gratings fabricated in tapered fiber [7630-76]

B. Li, Communication Univ. of China (China); H. Wei, Z. Tan, Beijing Jiaotong Univ. (China)

763020 Study on characteristics of optical bistable devices based on fiber Bragg grating [7630-20]

Y. Peng, K. Qiu, B. Wu, S. Ji, Univ. of Electronic Science and Technology of China (China)

$763021 \quad$ Multiwavelength erbium-doped fiber ring laser employing Fabry-Perot etalon [7630-196]

C. H. Yeh, Industrial Technology Research Institute (Taiwan, China); C. W. Chow, Y. F. Wu, F. Y. Shih, C. H. Wang, National Chiao Tung Univ. (Taiwan, China); S. Chi, Yuan Ze Univ. (Taiwan, China)

763022 Supercontinuum generation in tapered fibers [7630-70]

H. Li, L. Wei, X. Zhang, J. Tian, Y. Song, Beijing Univ. of Technology (China)

763023 Pole-zero diagram approach to the design of Michelson Gires-Tournois interferometer interleaver [7630-61]

J. Zhang, X. Yang, S. Yu, Shanghai Univ. (China)

763024 Frequency response of fiber-optic hydrophone with a novel mechanical anti-aliasing filter of side cavities [7630-04]

Z. Wang, Y. Hu, National Univ. of Defense Technology (China) 
763025 Widely tunable L-band Brillouin fiber laser incorporating a bismuth-based erbium-doped fiber [7630-111]

Y. Wei, B. Sun, T. Wang, Zhejiang Univ. (China) and Royal Institute of Technology (Sweden);

D. Chen, Zhejiang Normal Univ. (China) and Zhejiang Univ. (China)

763026 Femtosecond pulse compression in hollow-core photonic bandgap fibers [7630-84]

Y. Meng, S. Zhang, C. Jin, H. Li, X. Wang, Hebei Normal Univ. (China)

763027 Absolutely single polarization photonic crystal fiber based on a structure of sub-wavelength hole pitch [7630-59]

D. Chen, Zhejiang Normal Univ. (China) and Zhejiang Univ. (China)

763028 Study and fabrication of add/drop filter based on Bragg gratings reflection coupler [7630-36]

W. Jiang, L. Fan, Z. Liu, P. Tao, Beijing Jiaotong Univ. (China); J. Li, Beijing Jiaotong Univ. (China) and China Academy of Railway Sciences (China); S. Jian, Beijing Jiaotong Univ. (China)

763029 Growth of ultraviolet-induced $\mathrm{H}_{2}$-loaded long period fiber grating immediately after fabrication [7630-33]

J. Zheng, Y. Lu, W. Ren, L. Fan, Z. Liu, T. Ning, S. Jian, Beijing Jiaotong Univ. (China)

7630 2A Finite element analysis of the InP nano inner cladding fiber [7630-69]

Y. Duan, R. Zhang, P. Lang, Beijing Univ. of Posts and Telecommunications (China)

7630 2B Optimal design of birefringent Gires-Tournois optical interleaver [7630-15]

W. Wu, P. Liu, Wuhan Univ. of Technology (China)

$76302 \mathrm{C}$ Optimal control of light storage in atomic ensemble based on photon echoes [7630-77]

T. WU, Q. Chen, South China Univ. of Technology (China)

$76302 \mathrm{D} \quad$ Fiber Bragg grating sensors interrogation system using arrayed waveguide gratings demultiplexer [7630-14]

W. Wu, X. Liu, Wuhan Univ. of Technology (China)

$76302 \mathrm{E} \quad$ Bending sensor with tilted fiber Bragg grating interacting with multimode fiber [7630-113]

Y. Jin, China Jiliang Univ. (China); C. C. Chan, Nanyang Technological Univ. (Singapore);

$X$. Dong, China Jiliang Univ. (China); Y. F. Zhang, Nanyang Technological Univ. (Singapore)

$76302 \mathrm{~F} \quad \mathrm{C}+\mathrm{L}$ band multi-wavelength fiber laser based on cascaded semiconductor optical amplifier [7630-12]

Z. Wang, L. Zhang, D. Jia, Tianjin Univ. (China)

$76302 \mathrm{G}$ A novel technology to generate microwave signal based on multiple-frequency Brillouin fiber-ring laser [7630-26]

Y. Shen, PLA Univ. of Science and Technology (China) and Equipment Dept. of Army

(China); R. Wang, T. Pu, PLA Univ. of Science and Technology (China) 
$76302 \mathrm{H} \quad$ Novel technique for the measurement of photonic crystal fiber numerical aperture properties [7630-08]

Y. Han, L. Hou, Y. Guo, S. Li, X. Zhao, W. Wang, Yanshan Univ. (China); Z. Song, Liaoning Shihua Univ. (China)

$763021 \quad$ Influence of cores' shape on coupling length of dual-core fiber [7630-17]

J. Lin, Shanghai Univ. (China) and Guangxi Univ. (China); Z. Shi, Y. Bai, Q. Ge, Shanghai Univ. (China)

$76302 \mathrm{~J}$ Switchable dual-wavelength fiber laser based on PCF Sagnac loop and broadband FBG [7630-25]

W. Chen, S. Lou, S. Feng, L. Wang, H. Li, T. Guo, S. Jian, Beijing Jiaotong Univ. (China)

$76302 \mathrm{~K}$ Refractive sensitivity of mechanical long-period fiber grating in side-hole fiber [7630-07] Y. Jin, China Jiliang Univ. (China) and Nanyang Technological Univ. (Singapore);

C. C. Chan, Nanyang Technological Univ. (Singapore); H. Gong, X. Dong, China Jiliang Univ. (China)

Author Index 
Downloaded From: https://www.spiedigitallibrary.org/conference-proceedings-of-spie on 26 Apr 2023

Terms of Use: https://www.spiedigitallibrary.org/terms-of-use 


\title{
Organizing Committee
}

\author{
Honorary General Chairs
}

Guofan Jin, Tsinghua University (China)

Hequan Wu, Chinese Academy of Engineering (China)

Jie Zhang, Jiao Tong University (China)

Bingkun Zhou, Chinese Optical Society (China)

General Chairs

Kwok-Wai Cheung, The Chinese University of Hong Kong (Hong Kong, China)

Sailing He, Joint Research Center of the Royal Institute of Technology (Sweden) and Zhejiang University (China)

John Zyskind, JDSU Uniphase Corporation (United States)

Technical Program Chairs

Weisheng Hu, Shanghai Jiao Tong University (China)

Ming-Jun Li, Corning, Inc., (United States)

Dennis Matthews, University of California, Davis (United States)

Local Organizing Committee Chair

Yaohui Jin, Shanghai Jiao Tong University (China)

Local Organizing Committee

Nan Chi, Fudan University (China)

Weisheng Hu, Shanghai Jiao Tong University (China)

Feng Huang, Alcatel-Lucent Shanghai Bell (China)

Ronghui Qu, Institute for Optics and Fine Mechanics (China)

Weiqiang Sun, Shanghai Jiao Tong University (China) 
Downloaded From: https://www.spiedigitallibrary.org/conference-proceedings-of-spie on 26 Apr 2023

Terms of Use: https://www.spiedigitallibrary.org/terms-of-use 


\title{
Conference Committee
}

\author{
Conference Chair
}

Perry P. Shum, Nanyang Technological University (Singapore)

\section{Conference Cochairs}

C. Martijn de Sterke, University of Sydney (Australia)

David Richardson, University of Southampton (United Kingdom)

Ji Wang, Corning, Inc. (United States)

Tingyun Wang, Shanghai University (China)

Program Committee

Le Nguyen Binh, Monash University (Australia)

Zhuangqi Cao, Shanghai Jiao Tong University (China)

Christopher Caucheteur, Polytechnique de Mons (Belgium)

Xiangfei Chen, Nanjing University (China)

Zhihao Chen, Institute for Infocomm Research (Singapore)

Kin S. Chiang, City University of Hong Kong (Hong Kong, China)

Chi Wai Chow, National Chiao Tung University (Taiwan, China)

Youngjoo Chung, Gwangju Institute of Science and Technology

(Korea, Republic of)

Jon DeGroot, Dow Corning Corporation (United States)

Mable P. Fok, Princeton University (United States)

Claire Gu, University of California, Santa Cruz (United States)

Young-Geun Han, Hanyang University (Korea, Republic of)

John Harvey, The University of Auckland (New Zealand)

Helmut Heidrich, Fraunhofer Heinrich Hertz Institute (Germany)

Yicheng Lai, Data Storage Institute (Singapore)

Maryanne Large, University of Sydney (Australia)

Donghan Lee, Chungnam National University (Korea, Republic of)

Shenping Li, Corning, Inc. (United States)

Aoxiang Lin, Lehigh University (United States)

Gong-Ru Lin, National Taiwan University (Taiwan, China)

Deming Liu, Huazhong University of Science and Technology (China)

Xueming Liu, Xi'an Institute of Optics and Precision Mechanics (China)

Yunqi Liu, Shanghai University (China)

Jie Luo, Yangtze Optical Fiber and Cable (China)

Jean-Pierre Meunier, Université Jean Monnet (France)

Hai Ming, University of Science and Technology of China (China)

Jinyu Mo, Bookham Technology Co., Ltd. (China)

Kyunghwan Oh, Yonsei University (Korea, Republic of)

Namkyoo Park, Seoul National University (Korea, Republic of) 
Gang-Ding Peng, University of New South Wales (Australia)

Ching Eng Jason Png, A-STAR-Agency for Science, Technology, and

Research (Singapore)

Min Qiu, Royal Institute of Technology (Sweden)

Ronghui Qu, Shanghai Institute of Optics and Fine Mechanics, Chinese Academy of Sciences (China)

Yun-Jiang Rao, University of Electronic Science and Technology of China (China)

Akihiko Shinya, NTT Basic Research Laboratories (Japan)

Baishi Wang, Vytran LLC (United States)

Jian Wang, Huazhong University of Science and Technology (China)

Tomasz Woliński, Warsaw University of Technology (Poland)

Kenneth Kin-Yip Wong, University of Hong Kong (Hong Kong, China)

Kun Xu, Beijing University of Posts and Telecommunications (China)

Shinji Yamashita, University of Tokyo (Japan)

Min Yan, Technical University of Denmark (Denmark)

Changyuan Yu, National University of Singapore (Singapore)

Xuming Zhang, Hong Kong Polytechnic University (Hong Kong, China)

Yinian Zhu, Stevens Institute of Technology (United States)

\section{Session Chairs}

Nonlinear Optics

Xinyong Dong, Nanyang Technology University (Singapore)

Best Student Paper Competition

Martijn de Sterke, University of Sydney (Australia)

Optical Fiber

Benjamin J. Eggleton, University of Sydney (Australia)

Optical Pulses

Perry P. Shum, Nanyang Technological University (Singapore)

Polarization Effects and Measurements

Aoxiang Lin, Center for Optical Technologies, Lehigh University

(United States)

Photonic Crystals

Yong-Won Song, Korea Institute of Science and Technology

(Korea, Republic of)

Fiber Lasers

Shenping Li, Corning Incorporated (United States) 
Optical Processing

Aoxiang Lin, Center for Optical Technologies, Lehigh University (United States)

Fiber Gratings

John Canning, University of Sydney (Australia)

Optical Couplers

Perry P. Shum, Nanyang Technological University (Singapore)

Photonic Crystal Fibers I

Heike Ebendorff-Heidepriem, University of Adelaide (Australia)

Optical Waveguide Devices I

Wolfgang Sohler, University of Paderborn (Germany)

Fiber Design and Fabrication

Limin Tong, Sr., Zhejiang University (China)

Optical Waveguide Devices II

Yikai Su, Shanghai Jiao Tong University (China)

Photonic Crystal Fibers II

Boris T. Kuhlmey, CUDOS, University of Sydney (Australia)

Optical Amplifiers

John Ballato, Clemson University (United States)

Microstructured Fibers

Morten Ibsen, University of Southampton (United Kingdom)

Optical Devices I

Perry P. Shum, Nanyang Technological University (Singapore)

Optical Fiber

Ching Eng Jason Png, A-STAR-Agency for Science, Technology, and (Singapore)

Optical Devices II

Tao Lu, University of Victoria (Canada) 
Downloaded From: https://www.spiedigitallibrary.org/conference-proceedings-of-spie on 26 Apr 2023

Terms of Use: https://www.spiedigitallibrary.org/terms-of-use 